\title{
Detecting Misplaced RFID Tags on Static Shelved Items
}

\author{
Jiaqing Luo ${ }^{\dagger \ddagger}$ and Kang G. Shin ${ }^{\ddagger}$ \\ ${ }^{\dagger}$ School of Computer Science and Engineering, University of Electronic Science and Technology of China \\ ${ }^{\ddagger}$ Electrical Engineering and Computer Science, The University of Michigan — Ann Arbor, U.S.A. \\ csjluo@hotmail.com,kgshin@umich.edu
}

\begin{abstract}
A smart shelving system can visualize stock data in real time by leveraging item-level RFID tagging so that we can minimize out-of-stock and reduce warehousing and labor costs. The key issue of smart shelving is to locate RFID tags at any time, especially after misplacing tags. The detection of misplaced tags on stationary shelved items is very challenging due to position ambiguity, phase wrapping, device diversity, and phase ambiguity. Using a combination of theoretical analysis, simulation-based prediction and experimental verification, we propose an effective way of detecting misplaced tags, called FINDS, that integrates Particle Swarm Optimization (PSO), Synthetic Minority Over-sampling TEchnique (SMOTE) and Density-based Spatial Clustering of Applications with Noise (DBSCAN) algorithms to make theoretical and measured phases consistent with each other, and observe the phase shifts caused by misplaced tags. FINDS requires neither antenna movement nor external disturbances. We have implemented a prototype of FINDS with 20 tags and evaluated its performance, demonstrating FINDS's accuracy to be higher than 0.92 in the case of 2 stationary antennas.
\end{abstract}

\section{CCS CONCEPTS}

- Networks $\rightarrow$ Mobile networks.

\section{KEYWORDS}

RFID; misplaced tags; stationary tags; smart shelves

\section{ACM Reference Format:}

2019. Detecting Misplaced RFID Tags on Static Shelved Items. In The 17th Annual Int'l Conference on Mobile Systems, Applications, 8 Services (MobiSys '19), June 17-21, 2019, Seoul, Korea. ACM, New York, NY, USA, 13 pages. https://doi.org/10.1145/3307334. 3326085

\section{INTRODUCTION}

When implemented at the item level, RFID technology can help ensure the availability of the right product, on the right shelf, and at the right place, essentially all the time.

Permission to make digital or hard copies of all or part of this work for personal or classroom use is granted without fee provided that copies are not made or distributed for profit or commercial advantage and that copies bear this notice and the full citation on the first page. Copyrights for components of this work owned by others than ACM must be honored. Abstracting with credit is permitted. To copy otherwise, or republish, to post on servers or to redistribute to lists, requires prior specific permission and/or a fee. Request permissions from permissions@acm.org.

MobiSys '19, June 17-21, 2019, Seoul, Korea

(C) 2019 Association for Computing Machinery.

ACM ISBN 978-1-4503-6661-8/19/06 . .\$15.00

https://doi.org/10.1145/3307334.3326085
If a product is not on the shelf, customers often give up the purchase and shop it somewhere else. It is also more expensive to win back customers than keeping loyal customers in the store happy. Analysts estimate that the US retail industry loses approximately US $\$ 30$ billion annually due to products not being on the shelves [10]. Stock data feeds from a smart shelving system can help deliver more personalized, convenient customer service. Inventory replenishment can be managed based on real-time transactions data instead of forecasts based on previous sales during a similar period.

Smart shelving replaces visual checks by the store staff, and enables consistent shelf-space allocation compliance by automating shelf-level management of items placement and leveraging item-level tagging to ensure adherence to supplier shelf-space allocation. For example, tooth-brushes should always be placed adjacent to tooth-pastes to maximize crossselling opportunities.

The main challenge of smart shelving is to locate, in real time, passive tags. Prior work on locating tags - that includes determination of their relative and absolute positions - is either dynamic or static. The former requires to move RFID tags or reader antennas in order to monitor/acquire $\mathrm{RF}$ changes as a time series. On the other hand, the latter requires high-density reference tags for a priori collection of RSS distributions or an expensive infrastructure (i.e., consisting of multiple reader antennas or synthetic aperture radar) to analyze the tags' phase differences. Unfortunately, both of these approaches have practical limitations when used for the detection of misplaced tags in smart shelves. First, tags usually do not move, as items on shelves remain stationary for most of the time [20]. Second, it is very time-consuming and tedious to move reader antennas, since the scanning process usually needs to be continuous and stable [13, 17]. Third, the requirement of a complex and expensive infrastructure is unattractive/unsuitable for large-scale deployment and limits the horizontal expansion of shelves.

To overcome these limitations, we introduce a new costeffective and real-time detection scheme for tags in smart shelves, called FINDS (Find Items on Department store Shelves). We assume that tags are placed at fixed/predetermined positions on the shelves as is the case for garment racks or smart shelves, where the positions of hanging holes and shelving units are determined and fixed a priori. Although this assumption may limit application scenarios, it covers a large number of common use-cases and also facilitates FINDS's implementation and scalability. Our idea is to make the measured phase coincide with the theoretical phase, and identify the phase shifts caused by misplacement of tags. However, this is still challenging due to: (i) position ambiguity - 
the phase is periodic such that different positions may yield the same measured phase; (ii) phase wrapping — the measured phase may contain one or more $2 \pi$ jumps in order not to exceed its normal range $[0,2 \pi)$; (iii) device diversity the antennas may have different unknown initial phases even though they are of the same model; (iv) phase ambiguity the ImpinJ reader introduces a $\pi$ jump such that the reported phase can be the true phase or the true phase plus $\pi$.

We take three main steps to overcome these challenges: deployment optimization, phase unwrapping, and outlier detection. The deployment optimization maximizes the phase discrimination between any two positions by determining the optimal antenna positions, while the phase unwrapping provides an effective way of eliminating the effect of $2 \pi$ jumps by comparing the measured phase with the theoretical phase. Finally, the outlier detection estimates the systemic error to calibrate the measured phase, and detects abnormal phase deviations to identify misplaced tags.

FINDS has four salient features: (i) convenience - the setting of its parameters mainly depends on numerical calculations, facilitating easy deployment and management; (ii) scalability - it requires neither pre-collection of RF signals nor movement of tags/antennas; (iii) flexibility — it can dynamically adjust the number of antennas according to the number of tags; (iv) timeliness - it works stably and reliably even when the sampling time is small.

In summary, we make the following main contributions:

- Conducting a series of experiments to explore the challenges in detecting misplaced tags on stationary items;

- Development of an effective scheme for identifying misplaced tags, called FINDS, for smart shelving, with respect to position ambiguity, phase wrapping, device diversity, and phase ambiguity;

- Implementation a prototype of FINDS using ImpinJ R420 reader and Alien AZ-9346 tags; and

- Extensive evaluation of FINDS, demonstrating high accuracy ( $>0.85$ for 1 antenna and $>0.92$ for 2 antennas)

The rest of this paper is organized as follows. Section 2 discusses the related work, while Section 3 provides an overview of FINDS. Section 4 details FINDS and Section 5 evaluates FINDS's performance via extensive experimentation. Section 6 discusses the remaining issues and finally, the paper concludes with Section 7 .

\section{RELATED WORK}

A considerable amount of research has been done on RFIDbased indoor localization. Existing related work can be classified as dynamic tracking or static positioning.

\subsection{Dynamic Tracking}

Dynamic tracking collects time-varying RF signals by moving tags or antennas. Zhang et al. [23] presented a model of RSS dynamics to track transceiver-free objects. STPP [17] recognizes the relative positions of tags by observing sudden phase changes from different tags when the reader antenna is moved along a known direction. RF-Scanner [13] installs a
RFID reader on a moving robot to detect misplaced or laiddown books. OTrack [18] establishes a probabilistic model to recognize transient critical regions and monitors the order of tagged goods on an airport baggage carousel. Tagoram [22] leverages the phase of the backscattered signal, provided by a COTS RFID reader, for real-time tracking of mobile RFID tags. Wang et al. [19] proposed active and passive tracking of mobile antennas and tags, respectively. Both schemes were based on the Nelder-Mead nonlinear optimization that minimizes the error. Tagspin [6] emulates a circular antenna array by uniformly spinning on the edge of a rotating disk and designs a SAR-based method to pinpoint the target antenna. CBID [8] estimates Doppler shifts and RSS to detect and track tag movements and then infer the corresponding customer behaviors. TagBooth [14] uses RSS to exploit the motion of tagged commodities and utilizes phase to distinguish subtle customer actions. Tagwins [5] makes sense of mechanical rotation using dual tags' backscatter signals. It leveraged the relative signal of dual RFID tags to handle system shaking and proposed a compressive reading technique to recover the signal. RF-Dial [2] attaches a tag array to the surface of a specified object, and continuously tracks the translation and rotation of a tagged object. Katabi et al. [21] extracted multi-path profiles by the mobile antenna and adapted DTW to pinpoint a tag's location.

\subsection{Static Positioning}

Static positioning analyzes time-invariant RF signals with the help of reference tags or external disturbances. RADAR [1] utilizes the RSS gathered at multiple receiver locations to triangulate the user's locations. Triangulation is done with both empirically-determined and theoretically-computed RSS. LANDMARC [16] utilizes RSS to find $k$ nearest reference tags of an active target tag, and estimates the target's position. Hekimian-Williams et al. [9] exploited the phase difference between two receiving antennas for localization and tracking. BackPos [15] infers the differences of distance from the phases detected by antennas under a triangle constraint and employs hyperbolic positioning to narrow the tag's candidate positions. HMRL [20] leverages the humans' movements in a region to explore the order of tags in a $2 \mathrm{D}$ space.

Static positioning is much more difficult than dynamic tracking in which RF signals are a set of points rather than a group of sequences. Although prior static positioning approaches are fine-grained, they might be impractical to detect misplaced tags in smart shelves due to their poor scalability. Some fundamental issues of static positioning, such as phase wrapping and antenna diversity, have not yet been addressed.

\section{BACKGROUND AND OVERVIEW}

We first introduce the technical background of RF phase and then conduct a series of experiments to explore/identify challenges in RFID-based positioning with stationary devices. Finally, we present an overview of FINDS, the proposed detection of misplaced tags. 


\subsection{Background}

A passive RFID tag communicates via a backscatter radio link. The tag, without battery, harvests energy from the reader's signal. Let $d$ be the distance between the reader antenna and the tag, then the distance traveled by the signal will be $2 d$. The theoretical phase $\Theta$ can be calculated as:

$$
\Theta=2 \pi \frac{2 d}{\lambda}-2 K \pi,
$$

where $\lambda$ is the wavelength and $K$ an integer. The theoretical phase is a periodic function with period $2 \pi$ radians, which repeats every $\frac{\lambda}{2}$ in the distance between the tag and the reader antenna [11].

The measured phase $\theta$ includes a systemic error $\mu$, which is defined as

$$
\theta=2 \pi \frac{2 d}{\lambda}-2 k \pi+\mu .
$$

As specified in [11], $\theta$ ranges between 0 and $2 \pi$. Let $\mu=$ $\left(\theta_{T A G}+\theta_{A N T}\right) \bmod 2 \pi$, where $\theta_{T A G}$ and $\theta_{A N T}$ are the additional rotations representing the tag's reflection characteristics, the reader antenna's transmit and receive circuits, respectively.

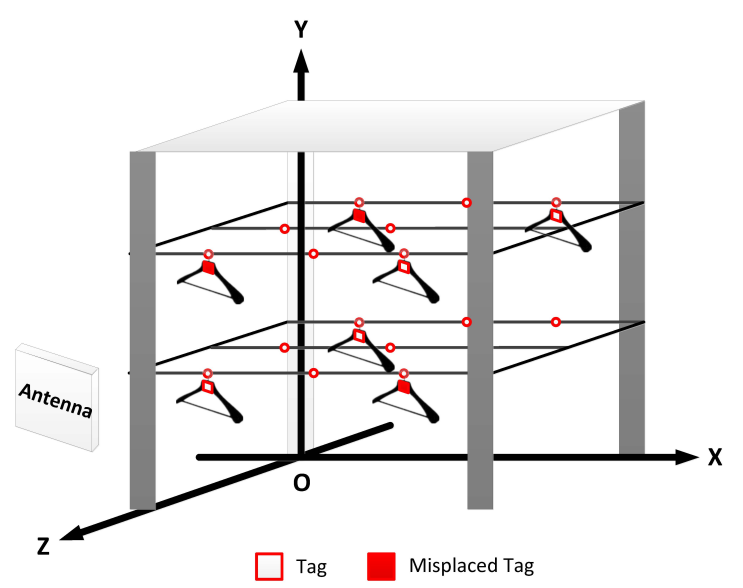

Figure 1: 3D shelf space

\subsection{Challenges}

We conducted a series of experiments with COTS ImpinJ readers and Alien tags. These experiments were designed to identify the potential difficulties in positioning tags with stationary devices. As shown in Fig. 1, we establish a 3D coordinate system according to the right-hand rule. The system is oriented counter-clockwise with respect to $z$-axis. Both tags and antennas are parallel to the $x y$-plane. For simplicity, we only deploy tags in the $x y$-plane. Note that the following experimental results are not limited to this $2 \mathrm{D}$ deployment of tags, as we observe the effect of the 3D distances between tag and antenna on the measured phases. Each tag is interrogated 200 times and the measured phase is extracted from the tag's reports. Prior research [15] has shown that the phase actually exhibits a stable statistical structure, which is preserved in the presence of frequency changes. For easy and clear observation, we chose a fixed frequency $921.875 \mathrm{MHZ}$, whose corresponding wavelength is $0.325 \mathrm{~m}$.

3.2.1 Position Ambiguity. Tags placed at different positions may yield the same measured phase $\theta$ due to the cycle slip of phase. According to Eq. (2), $\theta$ should repeat from 0 to $2 \pi$ every half wavelength $\left(\frac{\lambda}{2} \approx 0.163\right)$. We placed an antenna $A_{1}$ at $(0,0,1.2)$, and two tags $T_{1}$ and $T_{2}$ at $(-0.037,0.306,0)$ and $(-0.248,0.681,0)$. The distances from $A_{1}$ to $T_{1}$ and $T_{2}$ are $d_{1}^{1}=\left\|\overrightarrow{A_{1} T_{1}}\right\|=1.239$ and $d_{2}^{1}=\left\|\overrightarrow{A_{1} T_{2}}\right\|=1.402$, respectively. The difference of distance $\Delta d_{2,1}^{1}=d_{2}^{1}-d_{1}^{1}=0.162$ is very close to $\frac{\lambda}{2}$. Assuming that $\theta_{1}^{1}$ and $\theta_{2}^{1}$ are $T_{1}$ 's and $T_{2}$ 's measured phases from $A_{1}$, Fig. 2 shows that $\theta_{1}^{1}$ and $\theta_{2}^{1}$ almost overlap. The means of $\theta_{1}^{1}$ and $\theta_{2}^{1}$ are 1.782 and 1.777 , respectively.

We then placed another antenna $A_{2}$ at $(0,0.3,1.2)$. Distances from $A_{2}$ to $T_{1}$ and $T_{2}$ are $d_{1}^{2}=\left\|\overrightarrow{A_{2} T_{1}}\right\|=1.284$ and $d_{2}^{2}=\left\|\overrightarrow{A_{2} T_{2}}\right\|=1.485$, respectively. As Fig. 2 shows, one can distinguish $\theta_{1}^{2}$ from $\theta_{2}^{2}$. The means of $\theta_{1}^{2}$ and $\theta_{2}^{2}$ are 0.335 and 2.899 , respectively, as the distance difference $\Delta d_{2,1}^{2}=d_{2}^{2}-d_{1}^{2}=0.201$ is not an integer multiple of $\frac{\lambda}{2}$.

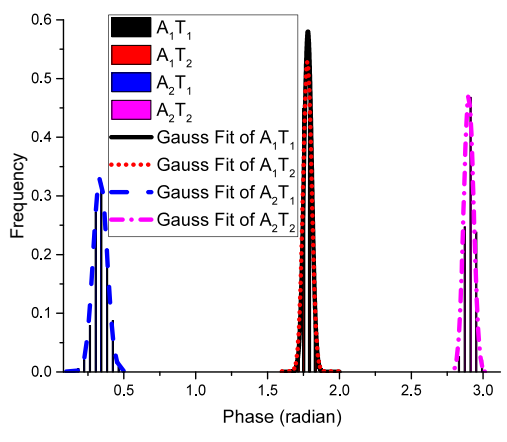

Figure 2: Position ambiguity

3.2.2 Phase Wrapping. Since the theoretical phase $\Theta$ has a normal range $[0,2 \pi)$, the measured phase $\theta=\Theta+\mu$ may exceed the range $[0,2 \pi)$ and contain one or more $2 \pi$ jumps. In such a case, $\theta$ will be wrapped around to stay within the normal range $[0,2 \pi)$. We placed an antenna $A_{1}$ at $(0.6,0.3$, $1.386)$ and 20 tags in a $4 \times 5$ array. [4, 24] suggest tags to be separated from each other by about $0.1 \mathrm{~m}$. If tags are too close to each other, they will suffer mutual coupling/interference effects. To reduce these effects, we separate two adjacent tags by $0.15 \mathrm{~m}$. Fig. 3 shows that $\theta$ does not vary with $\Theta$. Taking $T_{6}$ and $T_{8}$ as examples, $\theta_{6}^{1}-\Theta_{6}^{1}=4.429-2.045=2.383$, while $\theta_{8}^{1}-\Theta_{8}^{1}=4.786-0.455=4.331$. We calculate the Pearson correlation coefficient for $\theta$ and $\Theta$ to test whether there is a linear correlation between them. The coefficient of correlation is 0.429 , implying that there is a moderate linear correlation between $\theta$ and $\Theta$.

The $2 \pi$ jumps in the wrapped $\theta$ must be removed in order to make $\theta$ usable for further processing. The tag's $\theta$ presents 


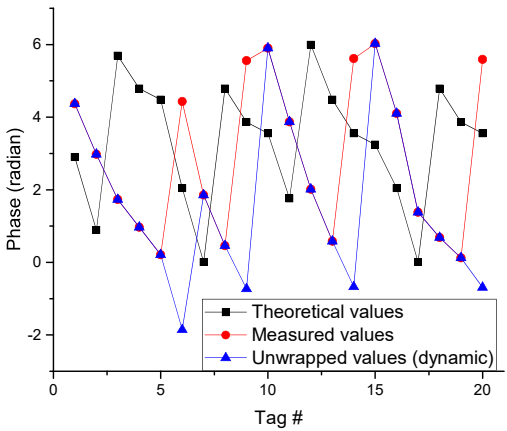

Figure 3: Phase wrapping
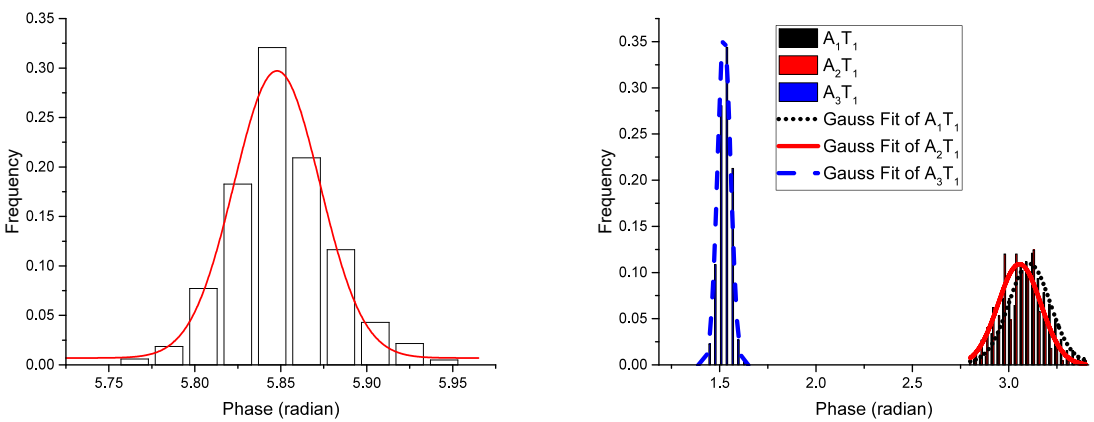

Figure 4: Tags of the same model Figure 5: Antennas of the same model a continuous form in the dynamic tracking. The existence of a wrap can be easily detected by the difference between two successive $\theta$ 's. If the difference is larger than $\pi$, then subtract $2 \pi$. If the difference is smaller than $-\pi$, then add $2 \pi$. Unfortunately, this phase unwrapping may not work correctly in the case of static positioning. Since both tags and antennas are placed at fixed positions, each tag's $\theta$ remains constant during observation. The difference between two successive $\theta$ 's will usually not exceed 0.1 radian [15]. So, in most cases, no phase wrap can be detected. We regard each tag's $\theta$ as a point rather than a time-series sequence, and attempt to unwrap all $\theta$ in a tag array. Let $\theta^{\prime}$ be the "unwrapped" $\theta$, then Fig. 3 indicates the non-existence of linear correlation between $\theta^{\prime}$ and $\Theta$. The coefficient of correlation is -0.13 . Using the unwrapping process, we can detect a phase wrap at $\theta_{6}^{1}$, because $\theta_{6}^{1}-\theta_{5}^{1}=4.429-0.208=4.221>\pi$. To correct $\theta_{6}^{1}$, we subtract $2 \pi$ and then get $\theta_{6}^{1^{\prime}}=-1.854$. However, we still find that $\theta_{6}^{1^{\prime}}-\Theta_{6}^{1}=-1.854-2.045=-3.899 \neq$ $\theta_{8}^{1^{\prime}}-\Theta_{8}^{1}=4.786-0.455=4.331$. The primary reason for the failure of unwrapping is that $\theta$ may be "under-sample". If the separation between two adjacent tags is too large, the difference between their $\theta$ 's may reach $\pi$ (or higher), and hence regarded incorrectly as a true phase wrap, when there is actually no real phase wrap. To the best of our knowledge, there has been no previous attempt to address this challenge with COTS RFID readers.

3.2.3 Device Diversity. Device diversity includes diversities of tags and the reader antennas. Prior research has shown device diversity to have a great effect on the phase measurement, thus leading to a natural question "can we eliminate this effect by choosing devices of the same model?" We first place an antenna at $(0,0.447,1.2)$ and 60 Alien AZ-9346 tags at $(0,0,0)$ in turn. The measured phase values are long-term averages. Fig. 4 shows that $\theta$ follows a Gaussian distribution with mean 5.85 and standard deviation 0.025 . This effect of the measurement error is negligible.

We deployed three Laird S9025PR antennas $A_{1}, A_{2}$ and $A_{3}$ at $(-0.3,0.073,1.2)$ in turn, and utilized them to interrogate $\operatorname{tag} T_{1}$ placed at $(0,0,0)$. These antennas are of the same model but from different batches. $A_{1}$ and $A_{2}$ 's batch numbers are 1316 while $A_{3}$ 's batch number is 1245 . Fig. 5 shows $\theta_{1}^{1}$ very close to $\theta_{1}^{2}$, while $\theta_{1}^{3}$ is very different from the other two. The means of $\theta_{1}^{1}$ and $\theta_{1}^{3}$ are shown to be 3.108 and 1.523 , respectively. We can also see that $A_{3}$ is more precise than $A_{1}$, since the standard deviation of $\theta_{1}^{3}$ is much smaller than that of $\theta_{1}^{1}$. These results indicate that even same-model antennas do not always yield consistent measurements. In order to improve system flexibility and scalability, we need to reduce the effect of antenna diversity.

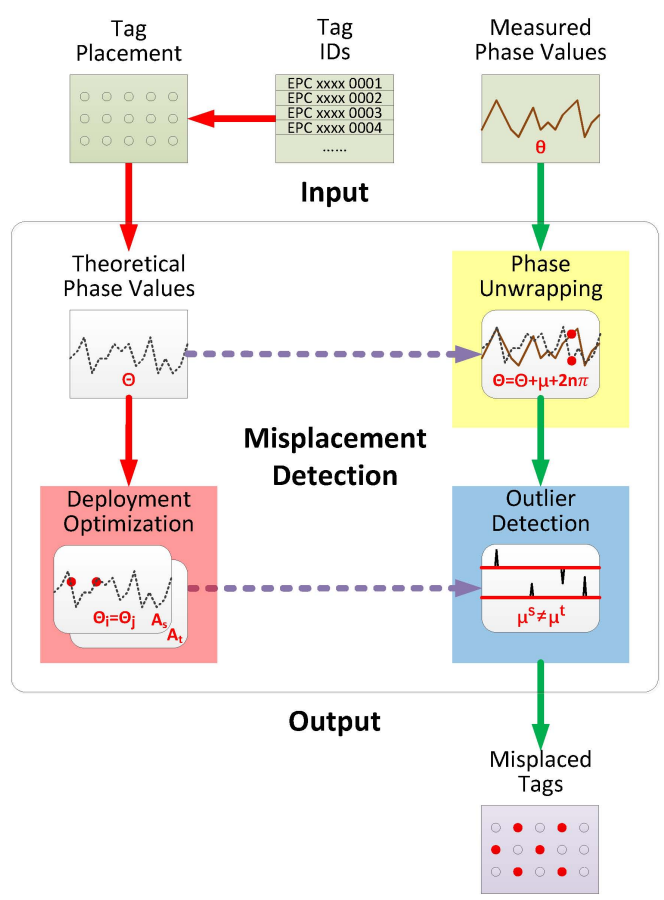

Figure 6: Basic FINDS framework

\subsection{Overview of FINDS}

The problem of detecting misplaced tags can be viewed as a special type of static positioning. We assume that tags are placed at a set of fixed positions as in clothing or unmanned shelves. Our basic idea is to calibrate the measured phases 
and detect the abnormal phase shifts caused by misplaced tags. FINDS neither collects signal fingerprints (RSS or phase) a priori nor tracks signal changes to handle a cold start, i.e., it does not assume all tags initially placed at the right place.

As shown in Fig. 6, FINDS consists of input, misplacement detection, and output. The input includes tag IDs, phase measurements, and tag placement. The misplacement detection consists of (antennas) deployment optimization, phase unwrapping, and outlier detection, which are designed to address the challenges identified in Section 3.2. The output is the tag IDs and the positions of misplaced tags.

Specifically, FINDS takes several steps to detect misplaced tags. First, it optimizes the deployment of antennas to reduce position ambiguities. It later collects tag IDs and phase measurements by interrogating tags. It then obtains the expected positions of tags from the pre-determined tag placement, followed by the calculation of theoretical phases corresponding to the expected positions. Next, it detects and corrects phase wraps according to a simple yet effective check, comparing the measured and the theoretical phases. Finally, it eliminates the effect of systemic errors and detects the anomalous phase shifts caused by misplaced tags.

\section{DETECTION OF MISPLACED TAGS}

We first describe how to detect misplaced tags and then present its technical details.

\subsection{Deployment Optimization}

Suppose there are $n_{A N T}$ antennas and $n_{T A G}$ tags, and let $\Theta_{i}^{s}$ be the theoretical phase of $\operatorname{tag} T_{i}\left(x_{i}, y_{i}, z_{i}\right)$ from antenna $A_{s}$ $\left(x^{s}, y^{s}, z^{s}\right)$, where $i \in\left\{1, \ldots, n_{T A G}\right\}$ and $s \in\left\{1, \ldots, n_{A N T}\right\}$. According to Eq. (1), $\Theta_{i}^{s}$ is determined by the distance between $A_{s}$ and $T_{i}, d_{i}^{s}=\left\|\overrightarrow{A_{s} T_{i}}\right\|$. Assuming that the antenna array topology is pre-fixed, let an arbitrary antenna $A_{r}$ $\left(x^{r}, y^{r}, z^{r}\right)$ be the reference point of the antenna array as shown in Fig. 7 . The position of $T_{i}$ relative to $A_{s}, \overrightarrow{A_{s} T_{i}}$ can then be written as:

$$
\begin{aligned}
\overrightarrow{A_{s} T_{i}} & =\overrightarrow{O T_{i}}-\overrightarrow{O A_{s}}=\overrightarrow{O T_{i}}-\overrightarrow{O A_{r}}-\overrightarrow{A_{r} A_{s}} \\
& =\left[\begin{array}{l}
x_{i} \\
y_{i} \\
z_{i}
\end{array}\right]-\left[\begin{array}{l}
x^{r} \\
y^{r} \\
z^{r}
\end{array}\right]-\left[\begin{array}{l}
x^{s}-x^{r} \\
y^{s}-y^{r} \\
z^{s}-z^{r}
\end{array}\right]
\end{aligned}
$$

The position of $T_{i}, \overrightarrow{O T_{i}}\left[x_{i}, y_{i}, z_{i}\right]^{\mathrm{T}}$, relates to the store layout, and thus usually remains unaltered. The position of $A_{s}$ relative to $A_{r}, \overrightarrow{A_{r} A_{s}}\left[x^{s}-x^{r}, y^{s}-y^{r}, z^{s}-z^{r}\right]^{\mathrm{T}}$, depends on the relative positions of antennas which also remains unaltered. Therefore, $\overrightarrow{A_{s} T_{i}}\left[x_{i}-x^{s}, y_{i}-y^{s}, z_{i}-z^{s}\right]^{\mathrm{T}}$ depends only on $\overrightarrow{O A_{r}}\left[x^{r}, y^{r}, z^{r}\right]^{\mathrm{T}}$. So, $\Theta_{i}^{s}$ can be viewed as a function of $\overrightarrow{O A_{r}}$, denoted by $\Theta_{i}^{s}\left(\overrightarrow{O A_{r}}\right)$.

Example: As shown in Fig. 7, we translate $A_{r}$ and $A_{s}$ without rotation. Since $A_{r}$ and $A_{s}$ are treated as a rigid body, if $A_{r}$ moves to $A_{r}^{\prime}$, then $A_{s}$ will be at $A_{s}^{\prime}$ such that $\overrightarrow{O A_{s}^{\prime}}=\overrightarrow{O A_{r}^{\prime}}+\overrightarrow{A_{r}^{\prime} A_{s}^{\prime}}=\overrightarrow{O A_{r}^{\prime}}+\overrightarrow{A_{r} A_{s}}$. Then, we have $\overrightarrow{A_{s}^{\prime} T_{i}}=$ $\overrightarrow{O T_{i}}-\overrightarrow{O A_{s}^{\prime}}=\overrightarrow{O T_{i}}-\overrightarrow{O A_{r}^{\prime}}-\overrightarrow{A_{r} A_{s}}$. As both $\overrightarrow{O T_{i}}$ and $\overrightarrow{A_{r} A_{s}}$ are

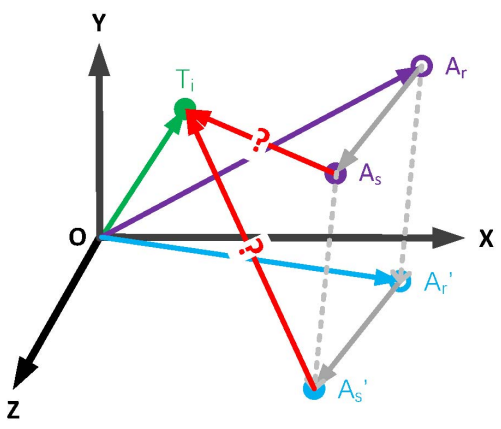

Figure 7: Antenna movement

unaltered, if $\overrightarrow{O A_{r}^{\prime}}$ is measured, then $\Theta_{i}^{s}\left(\overrightarrow{O A_{r}^{\prime}}\right)=\left(\frac{4 \pi}{\lambda} \| \overrightarrow{O T_{i}}-\right.$ $\left.\overrightarrow{O A_{r}^{\prime}}-\overrightarrow{A_{r} A_{s}} \|\right) \bmod 2 \pi$.

For any two arbitrary tags $T_{i}$ and $T_{j}$, if we exchange their positions, the amplitude of phase shift is $\Delta \Theta_{i, j}^{s}\left(\overrightarrow{O A_{r}}\right)=$ $\left|\Theta_{i}^{s}\left(\overrightarrow{O A_{r}}\right)-\Theta_{j}^{s}\left(\overrightarrow{O A_{r}}\right)\right|$ for each of them. Considering $n_{A N T}$ antennas, we define the phase shift resulting from the exchange of $T_{i}$ and $T_{j}, \Delta \Theta_{i, j}\left(\overrightarrow{O A_{r}}\right)$, as:

$$
\Delta \Theta_{i, j}\left(\overrightarrow{O A_{r}}\right)=\max \left\{\Delta \Theta_{i, j}^{s}\left(\overrightarrow{O A_{r}}\right)\right\},
$$

where $s \in\left\{1, \ldots, n_{A N T}\right\}$. In practice, we can select any two distinct tags and exchange their positions. There are $C_{2}^{n_{T A G}}$ such combinations. We enumerate $\Delta \Theta_{i, j}\left(\overrightarrow{O A_{r}}\right)$ for all pairs of tags, and define the minimum phase shift due to the tag misplacement, $\Delta \Theta\left(\overrightarrow{O A_{r}}\right)$, as:

$$
\Delta \Theta\left(\overrightarrow{O A_{r}}\right)=\min \left\{\Delta \Theta_{i, j}\left(\overrightarrow{O A_{r}}\right)\right\},
$$

where $i, j \in\left\{1, \ldots, n_{T A G}\right\}$. Then, $\Delta \Theta\left(\overrightarrow{O A_{r}}\right)$ is maximized to achieve the max-min fairness and avoid the worst results:

$$
{\overrightarrow{O A_{r}}}^{*}=\underset{\overrightarrow{O A_{r}}}{\arg \max } \Delta \Theta\left(\overrightarrow{O A_{r}}\right) .
$$

Finally, we utilize the Particle Swarm Optimization (PSO) algorithm [12] to find the optimal position of $A_{r}$.

Example: To investigate the optimal minimum phase shift $\Delta \overrightarrow{\Theta\left({\overrightarrow{O A_{r}}}^{*}\right)}$, we set the number of tags $n_{T A G}$ ranging from 10 to 30 and the number of antennas $n_{A N T}$ ranging from 1 to 3 . Two adjacent tags are separated by $0.15 \mathrm{~m}$ and two adjacent antennas by $0.45 \mathrm{~m}$. Fig. 8 shows that $\Delta \Theta\left({\overrightarrow{O A_{r}}}^{*}\right)$ increases with $n_{A N T}$, but decreases when $n_{T A G}$ increases. For example, $\Delta \Theta\left({\overrightarrow{O A_{r}}}^{*}\right)$ for $n_{T A G}=20$ increases by 1.34 radians when $n_{A N T}$ increases from 1 to 3 . On the other hand, $\Delta \Theta\left({\overrightarrow{O A_{r}}}^{*}\right)$ for $n_{A N T}=3$ decreases from 1.97 to 1.01 when $n_{T A G}$ increases from 10 to 30 .

\subsection{Phase Unwrapping}

The phase unwrapping is to restore a wrapped phase to a "correct" form that is free from $2 \pi$ jumps. Let $\theta_{i}^{s}$ be the measured phase of tag $T_{i}$ from antenna $A_{s}$ and $\mu_{i}^{s}$ be the systemic error caused by both tag $T_{i}$ and antenna $A_{s}$. We 


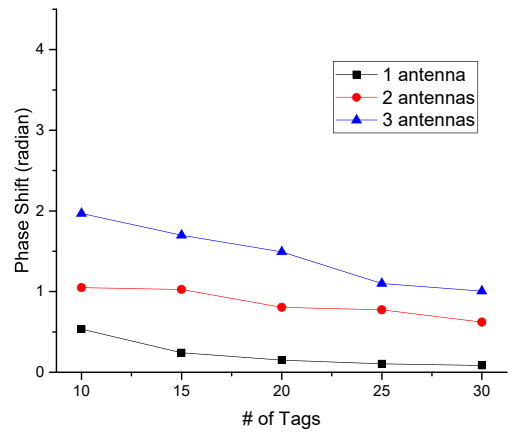

Figure 8: Optimal phase shift

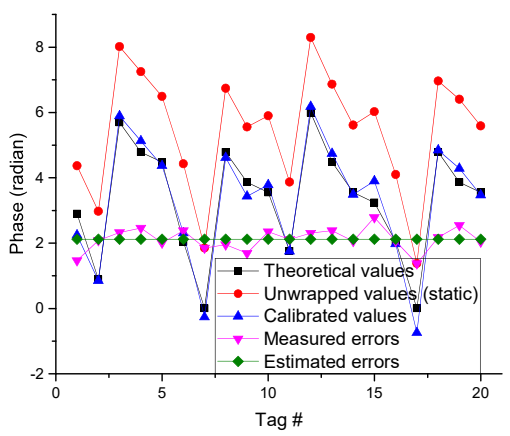

Figure 9: Unwrapping \& calibration

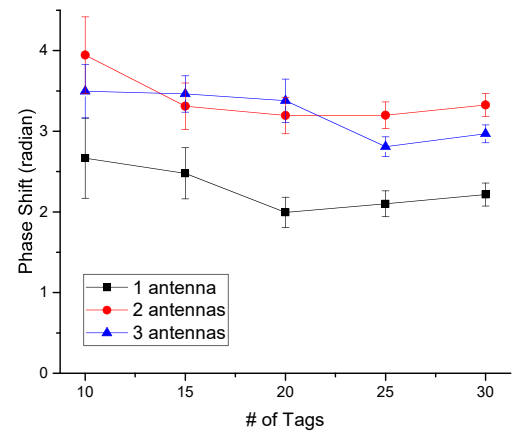

Figure 10: Phase shift subtract Eq. (1) from Eq. (2) to get:

$$
\Theta_{i}^{s}-\theta_{i}^{s}=2 \pi(k-K)-\mu_{i}^{s} .
$$

Since $\Theta_{i}^{s} \in[0,2 \pi)$ and $\theta_{i}^{s} \in[0,2 \pi)$, we have $\Theta_{i}^{s}-\theta_{i}^{s} \in$ $(-2 \pi, 2 \pi)$. Considering the sign of $\Theta_{i}^{s}-\theta_{i}^{s}$ leads to the following two cases.

Case 1: Assuming $\Theta_{i}^{s}-\theta_{i}^{s} \in(-2 \pi, 0]$, there are two subcases to consider: $\Theta_{i}^{s}-\theta_{i}^{s}>-2 \pi$ and $\Theta_{i}^{s}-\theta_{i}^{s} \leq 0$.

$$
\begin{aligned}
-2 \pi<\Theta_{i}^{s}-\theta_{i}^{s} \leq 0 & \Rightarrow\left\{\begin{array}{l}
2 \pi(k-K)-\mu_{i}^{s}>-2 \pi \\
2 \pi(k-K)-\mu_{i}^{s} \leq 0
\end{array}\right. \\
& \Rightarrow\left\{\begin{array}{l}
2 \pi(k-K)>\mu_{i}^{s}-2 \pi \geq-2 \pi \\
2 \pi(k-K) \leq \mu_{i}^{s}<2 \pi
\end{array}\right. \\
& \Rightarrow\left\{\begin{array}{l}
k-K>-1 \\
k-K<1
\end{array}\right. \\
& \Rightarrow k=K
\end{aligned}
$$

Case 2: Assuming $\Theta_{i}^{s}-\theta_{i}^{s} \in(0,2 \pi)$, there are two subcases: $\Theta_{i}^{s}-\theta_{i}^{s}>0$ and $\Theta_{i}^{s}-\theta_{i}^{s}<2 \pi$.

$$
\begin{aligned}
0<\Theta_{i}^{s}-\theta_{i}^{s}<2 \pi & \Rightarrow\left\{\begin{array}{l}
2 \pi(k-K)-\mu_{i}^{s}>0 \\
2 \pi(k-K)-\mu_{i}^{s}<2 \pi
\end{array}\right. \\
& \Rightarrow\left\{\begin{array}{l}
2 \pi(k-K)>\mu_{i}^{s} \geq 0 \\
2 \pi(k-K)<\mu_{i}^{s}+2 \pi<4 \pi
\end{array}\right. \\
& \Rightarrow\left\{\begin{array}{l}
k-K>0 \\
k-K<2
\end{array}\right. \\
& \Rightarrow k=K+1
\end{aligned}
$$

We can substitute for $k$ in Eq. (7) using Eqs. (8) and (9), yielding:

$$
\Theta_{i}^{s}= \begin{cases}\theta_{i}^{s}-\mu_{i}^{s} & \Theta_{i}^{s} \leq \theta_{i}^{s} \\ \theta_{i}^{s}+2 \pi-\mu_{i}^{s} & \Theta_{i}^{s}>\theta_{i}^{s}\end{cases}
$$

We define the unwrapped $\theta_{i}^{s}, \theta_{i}^{s^{\prime}}$, as:

$$
\theta_{i}^{s^{\prime}}= \begin{cases}\theta_{i}^{s} & \Theta_{i}^{s} \leq \theta_{i}^{s} \\ \theta_{i}^{s}+2 \pi & \Theta_{i}^{s}>\theta_{i}^{s} .\end{cases}
$$

According to Eq. (11), we unwrap $\theta_{i}^{s}$ as shown in Fig. 3. From Fig. 9, the change of $\theta_{i}^{s^{\prime}}$ is consistent with that of $\Theta_{i}^{s}$. The relation coefficient increases to 0.988 , implying a strong linear correlation between $\theta_{i}^{s^{\prime}}$ and $\Theta_{i}^{s}$. As $\theta_{i}^{s^{\prime}}=\Theta_{i}^{s}+\mu_{i}^{s}$, this result also indicates that $\mu_{i}^{s}$ is relatively stable.
Example: Let us consider $T_{6}$ and $T_{8}$ as an example. From Fig. 3, $\Theta_{8}^{1}=4.786>\theta_{8}^{1}=0.455$. By Eq. (11), we add $2 \pi$ and get $\theta_{8}^{1^{\prime}}=6.739$ and then we have $\theta_{6}^{1^{\prime}}-\Theta_{6}^{1}=4.429-2.045=$ $2.384 \approx \theta_{8}^{1^{\prime}}-\Theta_{8}^{1}=6.739-4.786=1.953$.

\subsection{Outlier Detection}

Our experimental results in Fig. 5 show that the antennas of the same model may have different initial phase rotations. Let us extend that to the more general case where different types of antennas are used. Before detecting misplaced tags, we estimate $\mu_{i}^{s}$ to calibrate $\theta_{i}^{s}$. We re-write Eq. (11) to calculate $\mu_{i}^{s}$ as:

$$
\mu_{i}^{s}=\theta_{i}^{s^{\prime}}-\Theta_{i}^{s}= \begin{cases}\theta_{i}^{s}-\Theta_{i}^{s} & \Theta_{i}^{s} \leq \theta_{i}^{s} \\ \theta_{i}^{s}+2 \pi-\Theta_{i}^{s} & \Theta_{i}^{s}>\theta_{i}^{s} .\end{cases}
$$

As we have already shown that $\mu_{i}^{s}$, albeit with some fluctuations, is stable, we can simplify the operation by deploying $n_{R E F}$ reference tags and define the estimated systemic error, $\hat{\mu^{s}}$, as:

$$
\hat{\mu^{s}}=\frac{1}{n_{R E F}} \sum_{j=1}^{n_{R E F}} \mu_{j}^{s},
$$

where $j \in\left\{1, \ldots, n_{R E F}\right\}$ and $n_{R E F} \ll n_{T A G}$. We replace $\mu_{i}^{s}$ with $\hat{\mu}^{s}$ and calibrate $\theta_{i}^{s}$ to approximate $\Theta_{i}^{s}$. The calibrated $\theta_{i}^{s}, \theta_{i}^{s "}$, can be defined as:

$$
\theta_{i}^{s ”}= \begin{cases}\theta_{i}^{s}-\hat{\mu^{s}} & \theta_{i}^{s} \geq \hat{\mu^{s}} \\ \theta_{i}^{s}+2 \pi-\hat{\mu^{s}} & \theta_{i}^{s}<\hat{\mu}^{s}\end{cases}
$$

Fig. 9 shows that $\theta_{i}^{s "}$ coincides with $\Theta_{i}^{s}$.

Suppose $A_{r}$ is at the optimal position and $\hat{\mu}^{s}$ is the real value. We consider two cases of $T_{i}$ : Case $1-$ if $T_{i}$ is at the right position then $\theta_{i}^{s "}-\Theta_{i}^{s}=\Theta_{i}^{s}-\Theta_{i}^{s}=0$; $\underline{\text { Case } 2}-$ if $T_{i}$ is

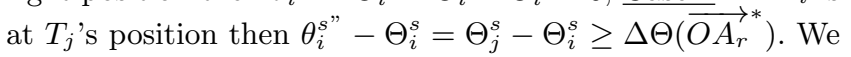
then define the estimated phase shift of $T_{i}, \Delta \hat{\Theta}_{i}$, as:

$$
\Delta \hat{\Theta}_{i}=\max \left\{\left|\theta_{i}^{s "}-\Theta_{i}^{s}\right|\right\}
$$

where $s \in\left\{1, \ldots, n_{A N T}\right\}$. Then, we detect the misplacement of $T_{i}$ by:

$$
\Delta \hat{\Theta}_{i} \geq \alpha \Delta \Theta\left({\overrightarrow{O A_{r}}}^{*}\right)
$$




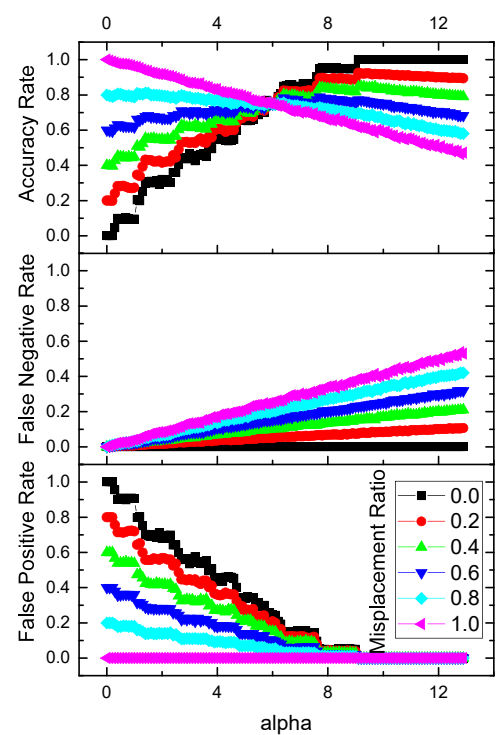

(a) 1 antenna

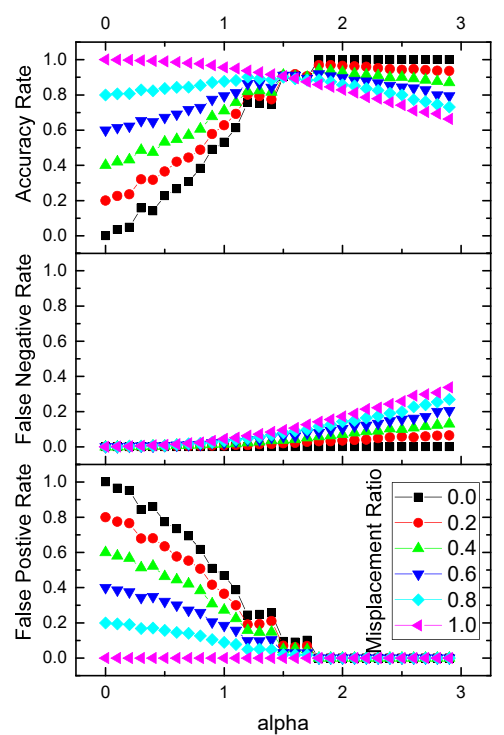

(b) 2 antennas

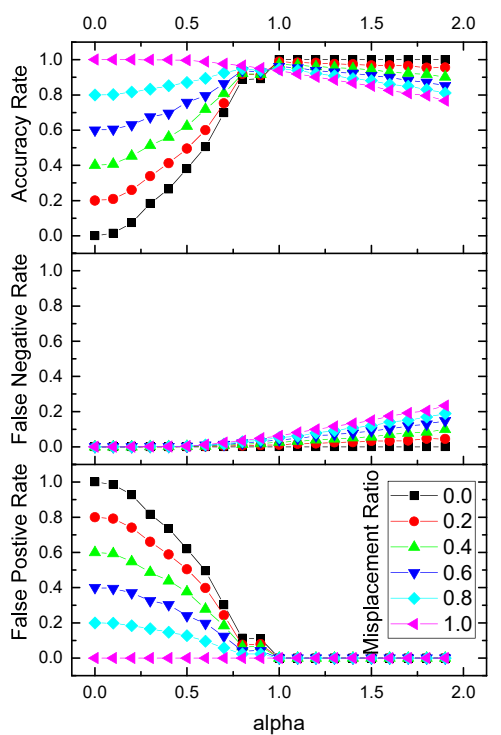

(c) 3 antennas

Figure 11: Coefficient

where $\alpha \Delta \Theta\left({\overrightarrow{O A_{r}}}^{*}\right)$ is the threshold for detecting misplaced tags, and $\alpha$ is the coefficient to control the false detection rate.

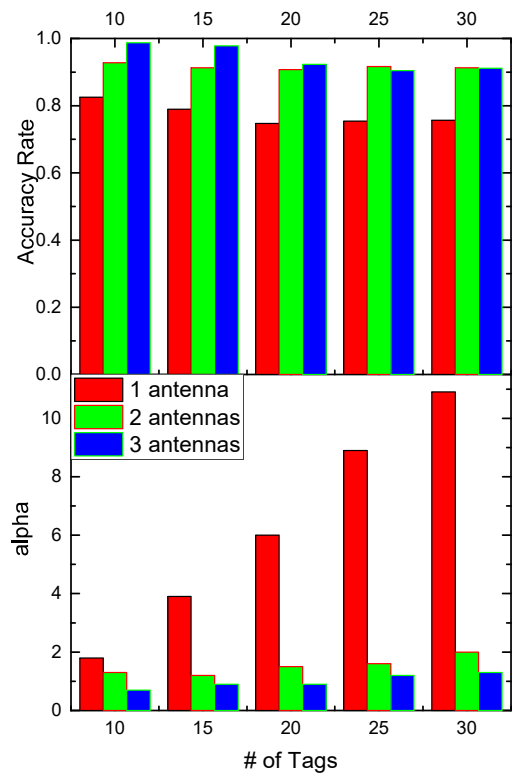

Figure 12: Coefficient vs. tags

\subsection{Parameter Setting}

We utilize both false positive rate, $f_{p}$, and false negative rate, $f_{n}$, to evaluate the detection accuracy. $f_{p}\left(f_{n}\right)$ is the number of false positives (negatives) divided by the total number of detections. The accuracy rate, $p$, can be calculated as $1-f_{p}-f_{n}$. Either $f_{p}$ or $f_{n}$ is caused by the error in estimating $\mu_{i}^{s}$, denoted as $\varepsilon_{i}^{s}$, which can be defined as $\mu_{i}^{s}-\hat{\mu^{s}}$. Such an error is difficult to predict due to its many causes such as sampling, measurement, and modeling errors. It is essential to choose an appropriate $\alpha$ to tolerate the uncertainty caused by $\varepsilon_{i}^{s}$.

Theoretically, we should set $\alpha$ within $(0,1]$ in order to cover the worst-case condition. In practice, $\Delta \Theta\left({\overrightarrow{O A_{r}}}^{*}\right)$ is very small when $n_{T A G}$ is large or $n_{A N T}$ is small. In such a case, we may need a larger $\alpha$ to balance between $f_{p}$ and $f_{n}$. Fig. 8 shows that $\Delta \Theta\left({\overrightarrow{O A_{r}}}^{*}\right)$ is only 0.15 for $n_{A N T}=1$ and $n_{T A G}=20$. Fortunately, the majority of $\Delta \Theta_{i, j}\left({\overrightarrow{O A_{r}}}^{*}\right)$ is much larger than $\Delta \Theta\left({\overrightarrow{O A_{r}}}^{*}\right)$. From Fig. 10, $\Delta \Theta_{i, j}\left({\overrightarrow{O A_{r}}}^{*}\right)$ has the mean of 1.99 (95\% CI: 1.81-2.18) for $n_{A N T}=1$ and $n_{T A G}=20$. In other words, the mean of $\Delta \Theta_{i, j}\left({\overrightarrow{O A_{r}}}^{*}\right)$, denoted as $\overrightarrow{\Delta \Theta_{i, j}\left({\overrightarrow{O A_{r}}}^{*}\right)}$, is $13 \times$ larger than $\Delta \Theta\left({\overrightarrow{O A_{r}}}^{*}\right)$, indicating the existence of room for adjusting $\alpha$.

Since $\varepsilon_{i}^{s}$ is unpredictable, we utilize the Synthetic Minority Over-sampling TEchnique (SMOTE) algorithm [3] to create "synthetic" $\varepsilon_{i}^{s}$, and conduct simulations to predict the effect of $\alpha$ on $p$. In particular, we generate the synthetic $\varepsilon_{i}^{s}$ as follows: (i) compute the error in each reference tag; (ii) compute the difference between errors under consideration and the nearest neighbors; (iii) randomly select a reference tag $T_{r}$ and choose one of its $k$ nearest neighbors, $T_{n n}$. Let $\varepsilon_{r}^{s}$ and $\varepsilon_{n n}^{s}$ be the errors in $T_{r}$ and $T_{n n}$, then the synthetic $\varepsilon_{i}^{s}$ can be generated by:

$$
\varepsilon_{i}^{s}=\varepsilon_{r}^{s}+\zeta\left|\varepsilon_{n n}^{s}-\varepsilon_{r}^{s}\right|,
$$


where $\zeta$ is a random number within $[0,1]$. With the synthetic $\varepsilon_{i}^{s}$, we can further generate synthetic $\theta_{i}^{s}$. If $T_{i}$ is placed at the right position, then $\theta_{i}^{s}$ " $=\Theta_{i}^{s}+\varepsilon_{i}^{s}$. If $T_{i}$ is at $T_{j}$ 's position, then $\theta_{i}^{s "}=\Theta_{j}^{s}+\varepsilon_{i}^{s}$.

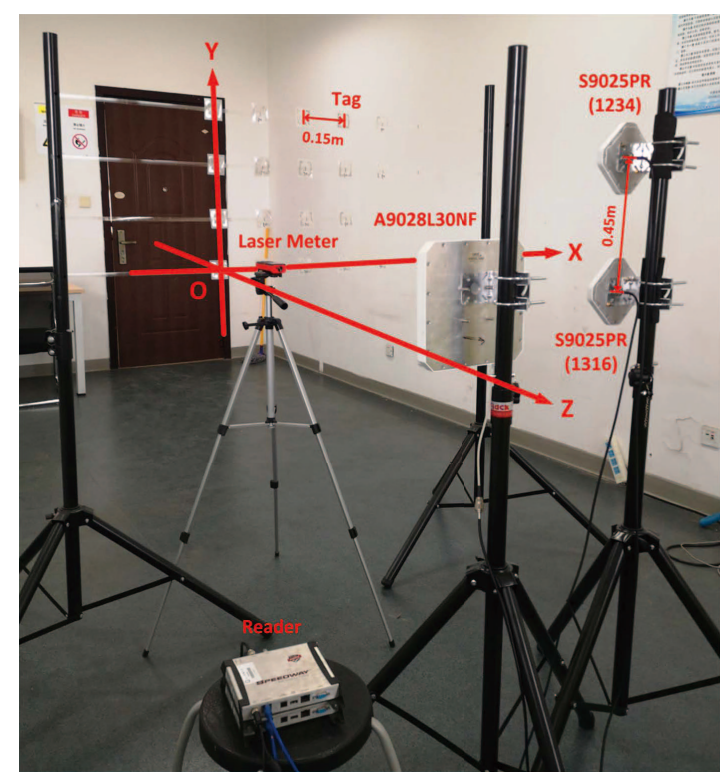

Figure 13: Experimental setup

In our simulation, we randomize the order of tags and define the misplacement ratio, $r$. This ratio is the number of misplaced tags divided by the total number of tags. The range of $\alpha$ is $\left(0,\left\lfloor\frac{\overrightarrow{\Delta \Theta_{i, j}\left({\overrightarrow{O A_{r}}}^{*}\right)}}{\Delta \Theta\left({\overrightarrow{O A_{r}}}^{*}\right)}\right\rfloor\right]$. At each step, $\alpha$ is incremented by 0.1 . The observation interval of the misplacement ratio $\Delta r$, is 0.2 . Figs. $11 \mathrm{a}-11 \mathrm{c}$ show that $f_{p}$ decreases and $f_{n}$ increases as $\alpha$ increases. $f_{p}$ is observed to decrease from 0.537 to 0.152 while $f_{n}$ increases from 0.008 to 0.048 for $r=0.4$ and $n_{A N T}=1$, when $\alpha$ increases from 1 to 6 . We also observe that the increase of $n_{A N T}$ helps reduce $f_{p}$. For $\alpha=1$ and $r=0.2, f_{p}$ decreases from 0.27 to 0 when $n_{A N T}$ increases from 2 to 3 . Another interesting observation is the existence of a balancing point where $p$ is insensitive to $r$. Such a point is $0.75,0.91$ and 0.92 for $n_{A N T}=1,2$ and 3 , respectively. The corresponding $\alpha$ is $6,1.5$ and 0.9 , respectively. Based on this observation, we calculate the variance of $p$ for different $r$ values. For given $n_{A N T}$ and $n_{T A G}, p$ is determined by $\alpha$, denoted by $p(\alpha)$. Let $\overline{p(\alpha)}$ be the expected value of $p(\alpha)$, then the optimal $\alpha, \alpha^{*}$, is defined as:

$$
\alpha^{*}=\underset{\alpha}{\arg \min }\left\{\Delta r \sum_{i=1}^{\frac{1}{\Delta r}}\left(p_{i}(\alpha)-\overline{p(\alpha)}\right)^{2}\right\} .
$$

We ran simulations to find $\alpha^{*}$ and its corresponding $\overline{p\left(\alpha^{*}\right)}$. Fig. 12 shows that $\alpha^{*}$ contributes to the stability of $\overline{p\left(\alpha^{*}\right)}$. As $n_{T A G}$ increases, $\overline{p\left(\alpha^{*}\right)}$ stays above $0.9\left(0.75-0.83\right.$ for $n_{A N T}=$ $1,0.91-0.93$ for $n_{A N T}=2$ and $0.91-0.99$ for $n_{A N T}=3$ ), although it decreases sightly. We have also observed that $\alpha^{*}$ increases dramatically from 1.8 to 10.9 when $n_{T A G}$ increases from 10 to 30 . The reason for this is that $\Delta \Theta\left({\overrightarrow{O A_{r}}}^{*}\right)$ becomes very small, as shown in Fig. 8. If $n_{T A G}$ keeps increasing, $\alpha$ will reach its maximum. In such a case, only larger $n_{A N T}$ can maintain the performance. We will in Section 5 verify the setting of $\alpha$ and compare the measured and the simulated results.

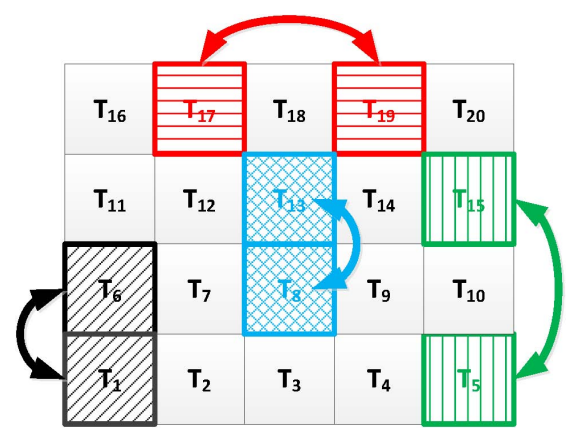

Figure 14: Tag placement

Because of the complexity of the environment, we recommend the adjustment of system parameters ( $\alpha$ and $\left.n_{A N T}\right)$ to improve the noise tolerance of FINDS. For example, the setting of $\alpha$ considers all possible cases of the misplacement $(r$ ranges from 0 to 1 ). However, in real situations, $r$ is unlikely to be greater than 0.5 . We can further increase $p$ or reduce $f_{p}$ by omitting rare cases.

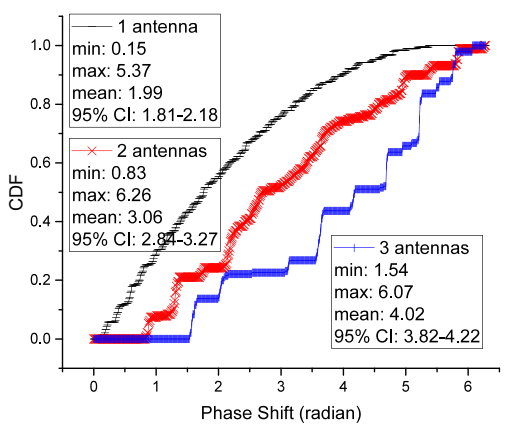

Figure 15: CDF

\section{EXPERIMENTAL EVALUATION}

We now describe the implementation and evaluation of a FINDS prototype.

\subsection{Implementation}

We implemented a prototype of FINDS using an ImpinJ reader in model R420, three Laird antennas in models A9028L30NF and S9025PR, and 20 Alien tags in model AZ-9346. The reader operates at $921.875 \mathrm{MHz}$ by default, and hence the wavelength is $0.325 \mathrm{~m}$. To account for device diversity, we 


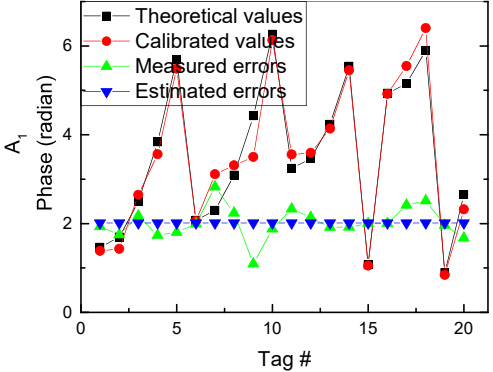

(a) 1 antenna

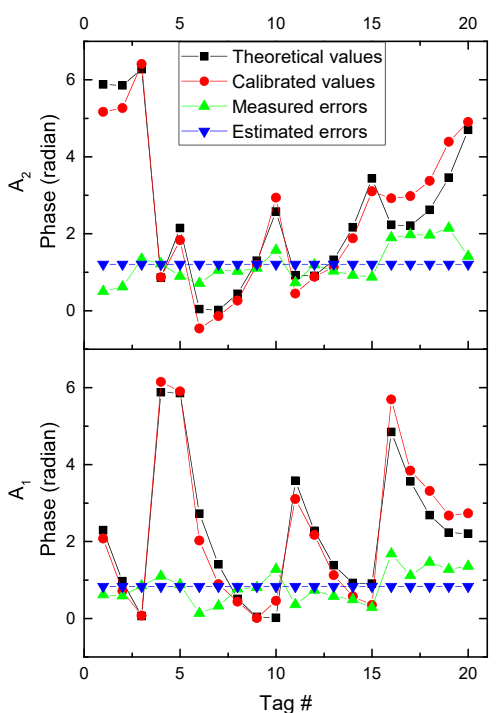

(b) 2 antennas

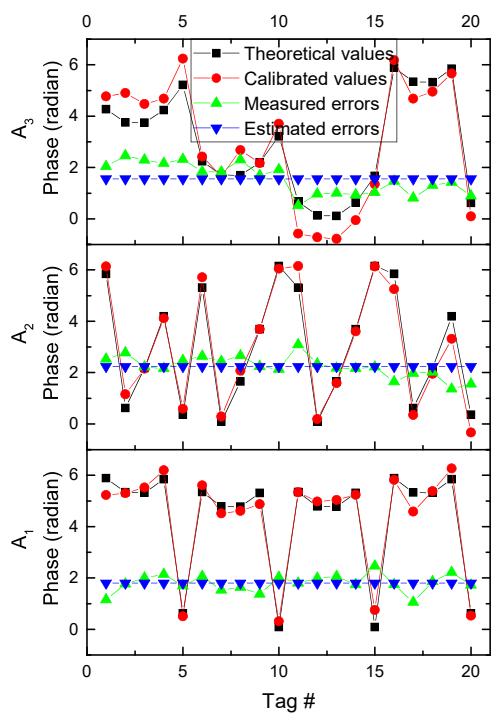

(c) 3 antennas

Figure 16: Calibration for $1-3$ antennas

choose a Laird A9028L30NF antenna and two Laird S9025PR antennas from different batches 1245 and 1316. During the measurement, the antennas and tags are viewed as the points located at their geometric centers.

As shown in Fig. 13, three antennas are separated by $0.45 \mathrm{~m}$ to form an L-shape array. Let $A_{1}$ be the reference antenna. Suppose $A_{1}$ 's coordinate is $\left(x^{1}, y^{1}, z^{1}\right), A_{2}$ 's and $A_{3}$ 's coordinates are $\left(x^{1}+0.45, y^{1}, z^{1}\right)$ and $\left(x^{1}, y^{1}+0.45, z^{1}\right)$, respectively. The antenna's transmission power is set to $30 \mathrm{~mW}$. We command the reader to immediately report phases and EPC numbers after a round of antenna scheduling. Fig. 13 also shows the deployment of 20 tags with spacing of $0.15 \mathrm{~m}$ in a $4 \times 5$ array. For ease of calculation, $T_{1}$ is assumed to be at $(0,0,0)$. The row-column layout of tags is shown in Fig. 14. Although we do not evaluate the performance of FINDS in the 3D tag deployment, we verify its effectiveness while varying antenna positions. The movement of antennas along the $z$-axis is equivalent to moving tags along the $z$-axis.

The distance between antenna and tag is measured with a distance laser meter with accuracy of $0.001 \mathrm{~m}$. The tag array's leveling accuracy is checked by a 2-line laser level.

\subsection{Evaluation}

Our experiments are designed to measure the false detection rate of misplaced tags, including deployment, unwrapping and detection.

5.2.1 Deployment. We optimize the deployment of antennas. Let $A_{1}\left(x^{1}, y^{1}, z^{1}\right)$ be the reference point of antennas. The PSO algorithm is used to find the optimal value of the objective function (Eq. (6)) subject to the constraints $x^{1} \in[0,0.6]$ $y^{1} \in[-0.3,0.3]$ and $z^{1} \in[1.5,3]$. These constraints need

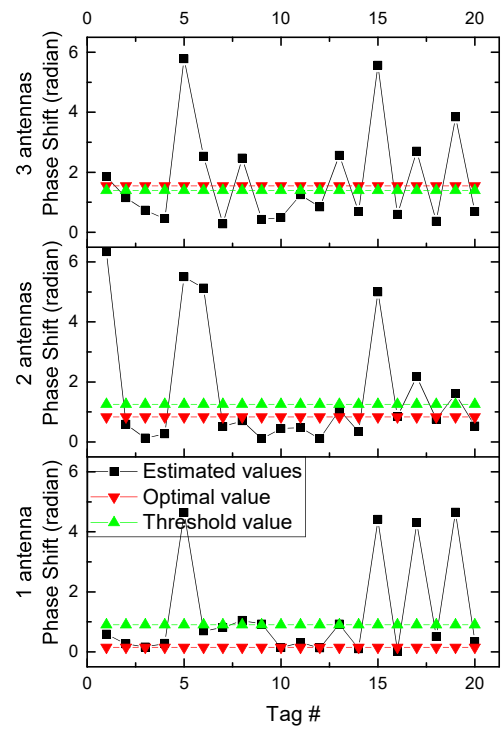

Figure 17: Detection to account for the range of reader antennas [15]. The optimal value ${\overrightarrow{O A_{1}}}^{*}$ is affected by the number of antennas $n_{A N T} .{\overrightarrow{O A_{1}}}^{*}$ is $[0.016,-0.084,1.5]^{\mathrm{T}},[0.532,-0.075,1.939]^{\mathrm{T}}$ and $[0.229,0.225,1.585]^{\mathrm{T}}$ for $n_{A N T}$ is 1,2 and 3 , respectively. Thus, $\Delta \Theta\left({\overrightarrow{O A_{r}}}^{*}\right)$ increases from 0.15 to 1.542 when $n_{A N T}$ increases from 1 to 3 . We plot the CDF of the phase shift caused by exchanging $T_{i}$ and $T_{j}, \Delta \Theta_{i, j}\left({\overrightarrow{O A_{r}}}^{*}\right)$, in Fig. 15 . There are $C_{2}^{20}=190$ combinations of 2 tags out of 20 tags. The mean of $\Delta \Theta_{i, j}\left({\overrightarrow{O A_{r}}}^{*}\right)$ is 1.99 (95\% CI: 1.81-2.18), 3.05 


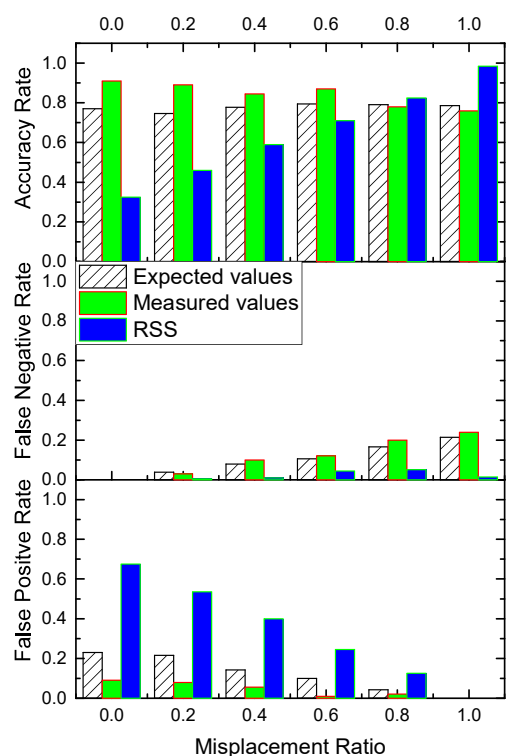

(a) 1 antenna

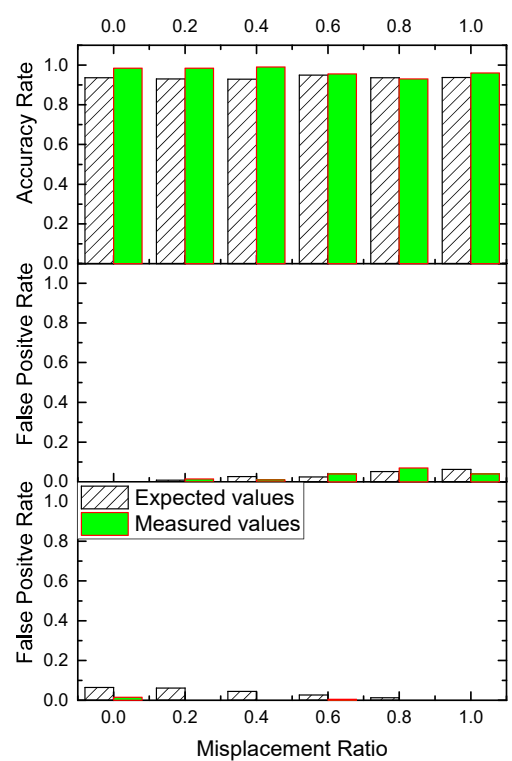

(b) 2 antennas

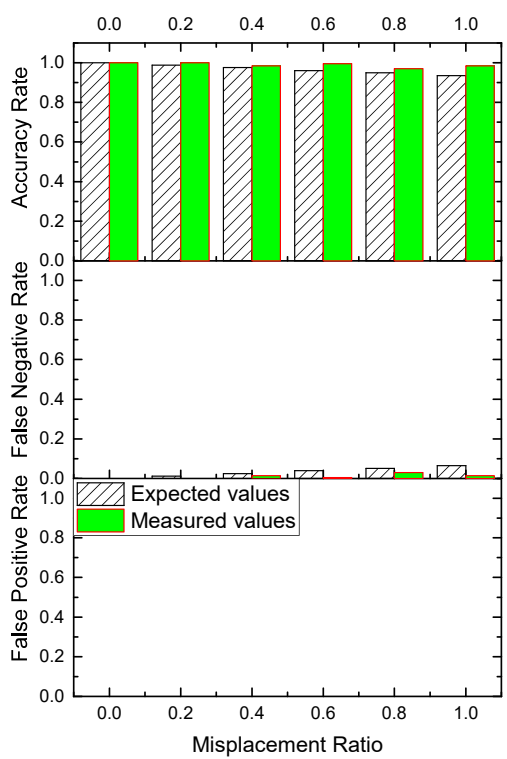

(c) 3 antennas

Figure 18: Verification with 1-3 antennas

(95\% CI: 2.84-3.27), and 4.02 (95\% CI: 3.82-4.22) for $n_{A N T}$ is 1,2 and 3 , respectively.

5.2.2 Unwrapping. We unwrap and calibrate the measured phase. After optimally deploying antennas, we measure the phase of each tag. A tag is interrogated 200 times and the average is recorded. Then, we unwrap the measured phase values by using Eq. (11). We randomly select 5 of 20 tags as reference tags. We use Eqs. (13) and (14) to estimate the systemic errors and calibrate the measured phase values. Figs. 16a-16c show that the measured systemic error is relatively stable, albeit with some fluctuations. As described in Section 4.4, these fluctuations are the main culprit for false detections. Considering the case of $n_{A N T}=2$, the maximum and minimum values of the measured systemic error related to $A_{1}$ are $\mu_{16}^{1}=1.68$ and $\mu_{6}^{1}=0.13$, respectively. The possible reason for this error is that $T_{16}$ is farther away from $A_{1}$ 's geometrical centerline than $T_{6}$. The EM may be emitted from $A_{1}$ 's edge, instead of its geometrical center, yielding a larger measurement error [15]. Another interesting observation is that $A_{s}$ 's estimated systemic error, $\hat{\mu}^{s}$, may vary with the position of $A_{s}$. For example, $\hat{\mu}^{1}$ changes from 0.84 to 1.81 when $A_{1}$ moves from $(0.532,-0.075,1.939)$ to $(0.229,0.225,1.585)$. This observation suggests that $\hat{\mu}^{1}$ might not be predictable due to the measurement error.

5.2.3 Detection. We first detect phase outliers. As shown in Fig. 14, we exchange 4 pairs of tags $\left\{T_{1}, T_{6}\right\},\left\{T_{5}, T_{15}\right\}$, $\left\{T_{6}, T_{13}\right\}$ and $\left\{T_{17}, T_{19}\right\}$. We detect misplaced tags by Inequality (16) and set the coefficient $\alpha$ to 1 . For example, we can catch $T_{5}$ when $n_{A N T}=3$ because $\Delta \hat{\Theta}_{5}=5.78>$ $\Delta \Theta\left({\overrightarrow{O A_{r}}}^{*}\right)=1.54$. Fig. 17 shows that all misplaced tags can be detected when $n_{A N T}=3$, while missing $T_{8}$ when $n_{A N T}=2$. We also notice that $T_{16}$, placed at the right position, is regarded as misplaced when $n_{A N T}=2$ due to $\Delta \hat{\Theta}_{16}=0.85>\Delta \Theta\left({\overrightarrow{O A_{r}}}^{*}\right)=0.83$, which turns out to be a false positive. The possibility of false positives may increase as $n_{A N T}$ decreases. There are 4 negatives, $T_{7}, T_{9}, T_{11}$ and $T_{20}$, that yield positive test outcomes when $n_{A N T}=1$. We can reduce the false positive rate, $f_{p}$, by using $\alpha^{*}$ obtained from simulation. As shown in the figure, $f_{p}$ decreases to 0 if $\alpha$ is set to the optimal value, 6 . However, this is achieved at the cost of increasing the false negative rate $f_{n}$ from 0 to 0.15 .

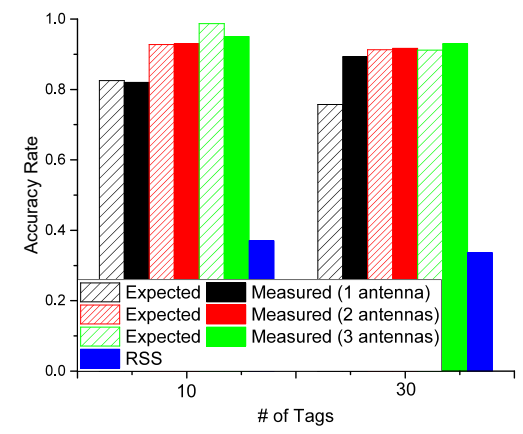

Figure 19: Accuracy vs. \# of tags

Let us verify the parameter setting. As suggested in Section 4.4, if $n_{T A G}=20$, then $\alpha^{*}$ is $6,1.5$ and 0.9 for $n_{A N T}=1$, 2 and 3 , respectively. The misplacement ratio $r$ increases from 0 to 1 with an increment of 0.2 . For each increment of 
$r$, we repeat the experiment 10 times and randomly shuffle tags each time. Figs. 18a-18c show the accuracy ratio $p$ under various conditions. Overall, the measured values of $p$ adequately fit the expected values of $p$. For $n_{A N T}=1$ and $r=0.2$, the measured value of $p$ achieves 0.89 , which is higher by 0.15 than the expected value. When $n_{A N T}=2$ or 3 , then $f_{p}$ is virtually invisible. Fig. $18 \mathrm{c}$ shows $f_{p}=0$ $\forall r$. The trend of the change of $f_{n}$ is opposite to that of $f_{p}$. Fig. 18a shows that $f_{n}$ increases from 0.03 to 0.2 when $r$ increases from 0.2 to 0.8 . We also compare FINDS with the RSS-based fingerprinting (RSS). Fig. 18a shows FINDS to outperform RSS in the majority of cases. For $r=0.2, p$ of FINDS is almost twice that of RSS. In fact, RSS might not be reliable for positioning static tags, if the centimeter precision is required. Similar results have also been reported in [20].

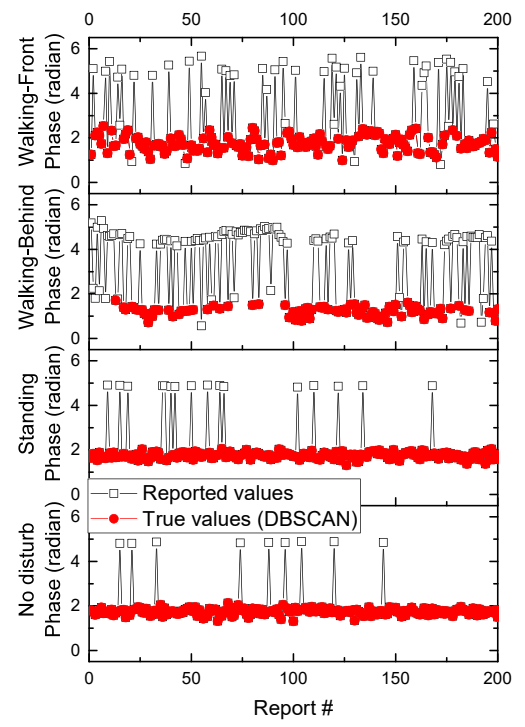

Figure 20: Effect of disturbances

We now vary the number of tags. We set $r$ to 0.2 and $n_{T A G}$ to 10 and 30 . As already shown in Fig. 12, when $n_{T A G}$ increases from 10 to $30, \alpha^{*}$ is 1.8 and 0.7 for $n_{A N T}=1 ; 6$ and 0.9 for $n_{A N T}=2$; and 10.9 and 3 for $n_{A N T}=3$. We randomly shuffle tags before each experiment. Fig. 19 shows that $p$ of FINDS is $0.93,1$ and 0.92 for $n_{A N T}=2$ when $n_{T A G}$ increases from 10 to 30 , which is almost $3 \mathrm{x}$ higher than that of RSS. These results suggest that the increase of $n_{T A G}$ has little effect on $p$ if $\alpha$ is selected properly.

\subsection{Improvement}

We can improve FINDS further by considering additional factors, such as human-activity-induced disturbances and sampling time/duration before detection.

5.3.1 Disturbances. FINDS is always running in the background, assuming that both tags and antennas are stationary. We introduce three types of disturbances, which do not include shoppers' activities, such as picking up and turning

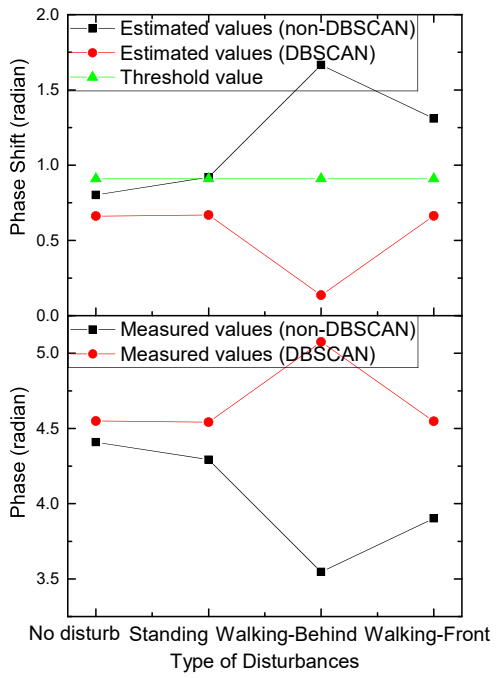

Figure 21: Resolution of phase ambiguity

items: (i) Standing - a person stands about $0.1 \mathrm{~m}$ behind the tag array; (ii) Walking-Behind - a person walks back and forth behind the tag array; (iii) Walking-Front - a person walks back and forth in front of the tag array. The parameters used in this experiment are $n_{A N T}=1, n_{T A G}=20$ and $\alpha=\alpha^{*}=6$. The placement of tags is the same as that in Fig. 14. We randomly select a tag $T_{9}$ and examine its reports in $\log$ files. We would like to point out that the reported phase, denoted as ' $\theta_{9}^{1}$, is not the same as the measured phase $\theta_{9}^{1}$ [15]. To obtain $\theta_{9}^{1}$, we need to change $\theta_{9}^{1}=2 \pi-^{\prime} \theta_{9}^{1}$. Fig. 20 shows that ${ }^{\prime} \theta_{9}^{1}$ fluctuates wildly under human-activityinduced disturbances, especially when a person walks nearby tags. The phase variation is mostly due to phase ambiguity and frequency shift.

Phase ambiguity is introduced by the device. As described in the low-level user data support for ImpinJ readers [11], the reader's processing of received signal introduces a $\pi$ jump such that the reported phase can be the true phase or the true phase plus $\pi$. As shown in Fig. 20, ' $\theta_{9}^{1}$ for No Disturb is either 1.7 or 4.8 . This figure also illustrates that phase ambiguity is much more severe under dynamic disturbances (Walking-Behind and Walking-Front) than under static disturbance (Standing). In practice, we may regard the true phase not simply as the minimum due to the phase wrapping described in Section 3.2.2. For example, if the true phase is $\pi$, we expect to obtain two values $\pi$ and $2 \pi$, but there could be three or more values, because a value near $2 \pi$ may flip to 0 due to the effect of thermal noise. It would become even more complicated if the true phase is close to $2 \pi$. In such a case, $2 \pi$ is neither minimum nor majority. To eliminate this ambiguity, we utilize the Density-based Spatial Clustering of Applications with Noise (DBSCAN) algorithm [7] to find the most common phase. Such an algorithm contains a pre-processing procedure for unwrapping phases near boundaries. In particular, if both 0 and $2 \pi$ are reported, the minority is wrapped. Fig. 20 


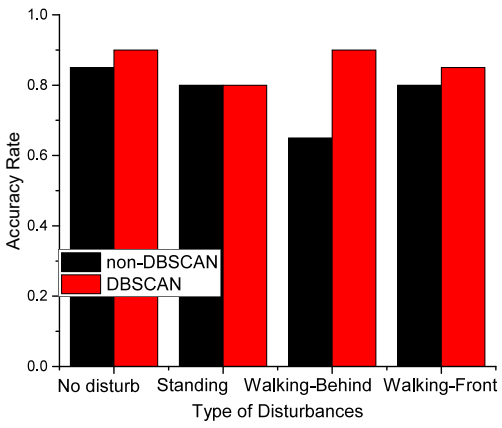

Figure 22: Robustness to disturbances

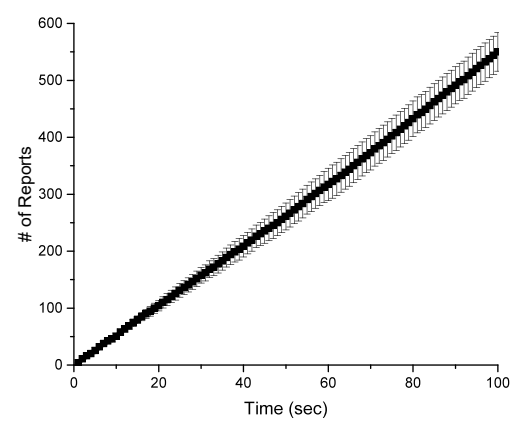

Figure 23: \# of reports vs. time

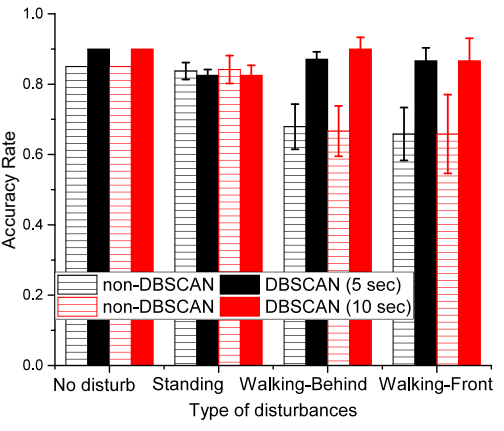

Figure 24: Sampling time shows that DBSCAN is effective in picking the true $\theta_{9}^{1}$. After resolving the phase ambiguity, FINDS can obtain a relatively stable $\theta_{9}^{1}$. Fig. 21 illustrates that $\theta_{9}^{1}$ shifts 0.51 and 0,01 for non-DBSCAN and DBSCAN, respectively, in the case of Walking-Front. As shown in the figure, DBSCAN improves FINDS's capability of resisting human-activity-induced disturbances. The estimated phase shift $\Delta \hat{\Theta}_{9}$ for non-DBSCAN is higher than the threshold $\alpha^{*} \Delta \Theta\left({\overrightarrow{O A_{r}}}^{*}\right)=6 \times 0.151=0.91$ in the presence of disturbance, while that for DBSCAN is always lower than the threshold in all cases.

The shift of the resonance frequency is caused by nearby objects. As shown in Fig. 21, in case of Walking-Behind, $\theta_{9}^{1}$ still shifts 0.53 even though the phase ambiguity has been resolved. The effect of frequency shift is difficult to eliminate, but FINDS can reduce its sensitivity to errors by deploying more antennas.

Overall, FINDS can work in complex environments. Fig. 22 shows that, although only one antenna is used, $p$ of FINDS with DBSCAN remains above 0.8 in all cases.

5.3.2 Sampling Duration/Time. FINDS is designed to detect misplaced tags in real time, i.e., it has a very short sampling time before each detection. We count the number of reports from each tag within 100 seconds. Fig. 23 shows that the number of reports increases linearly with the sampling time. On average, the number of reports increases at the rate of 5 per second. We set the sampling time to 5 and 10 seconds, and continuously observe $p$ within 60 seconds. Fig. 24 shows the limited effect of sampling time on the mean $p$. Under the same condition of DBSCAN not being used and WalkingBehind, the mean of $p$ increases slightly from 0.67 to 0.68 when the sampling time decreases from 10 to 5 seconds. The possible reason for this is that $\pi$ jumps appear randomly during sampling.

Another notable observation is that DBSCAN not only increases the mean of $p$, but also decreases the variance of $p$. Under dynamic disturbances, the standard deviation of $p$ for DBSCAN is a half of that for non-DBSCAN.

\section{DISCUSSION}

Our motivation for removing the inconsistency between measured and theoretical phases is to evaluate and optimize the performance of FINDS through numerical calculations. Some basic principles and algorithms of FINDS can also be used in other applications. For instance, we can deploy some reference tags at known positions, and then estimate systemic errors by the unwrapping, which could be helpful for positioning or tracking tags at unknown positions.

There still exist some uncertainties in FINDS (as our experimental results showed) that need to be investigated further: (i) measurement error - a small angular deviation may result in a large distance measurement error; (ii) inter-tag interference - a phase shift occurs when tags are placed too close to each other; (iii) reference tags - the number and positions of reference tags may affect the estimation of systemic errors; (iv) multi-path effects - shelves made of metal or backed against a wall may have a strong scattering.

In future, we would like to use a tag grid, which is similar to the Amsler grid used in eye examinations, for detecting and calibrating the phase shifts caused by distance measurement errors.

\section{CONCLUSION}

In this paper, we have first identified and addressed the challenges in positioning static tags, and then developed a simple yet practical approach, FINDS, for detecting misplaced tags in smart shelves. FINDS is more efficient and scalable than the state-of-the-art, as it requires only a few stationary antennas. We have also implemented a prototype of FINDS with COTS RFID products and conducted comprehensive experiments. Our evaluation has shown FINDS to achieve $>$ 0.92 accuracy only with 2 antennas. Thus, FINDS has great potential for various RFID applications.

\section{ACKNOWLEDGMENTS}

Jiaqing Luo was supported in part by the National Natural Science Foundation of China under Grant 61602093, the Key Project of Science and Technology of Sichuan under Grants 2018FZ0099 and 18ZDYF2554 and the Fundamental Research Funds for the Central Universities under Grants ZYGX2018J053 and ZYGX2018J055.

Kang Shin was supported in part by the US National Science Foundation under Grant CNS-1646130. 


\section{REFERENCES}

[1] P. Bahl and V. N. Padmanabhan. 2000. RADAR: An Inbuilding RF-based User Location and Tracking System. In INFOCOM 2000: Proceedings of the 19th Annual IEEE International Conference on Computer Communications, Vol. 2. 775-784.

[2] Yanling Bu, Lei Xie, Yinyin Gong, Chuyu Wang, Lei Yang, Jia Liu, and Sanglu Lu. 2018. RF-Dial: an RFID-based 2D HumanComputer Interaction via Tag Array. In INFOCOM 2018: Proceedings of the 37th IEEE International Conference on Computer Communications. Honolulu, HI, USA.

[3] Nitesh V. Chawla, Kevin W. Bowyer, Lawrence O. Hall, and W. Philip Kegelmeyer. 2002. SMOTE: Synthetic Minority Over sampling Technique. Journal of Artificial Intelligence Research 16,1 (2002), 321-357.

[4] DoD. 2014. United States Department of Defense Suppliers Passive RFID Information Guide, Version 15.0. https://www.acq.osd $\mathrm{mil} / \mathrm{log} / \mathrm{sci} /$ rfid_FAQs.html

[5] Chunhui Duan, Lei Yang, Huanyu Jia, Qiongzheng Lin, Yunhao Liu, and Lei Xie. 2018. Robust Spinning Sensing with Dual-RFIDTags in Noisy Settings. In INFOCOM 2018: Proceedings of the 37th IEEE International Conference on Computer Communications. Honolulu, HI, USA.

[6] C. Duan, L. Yang, Q. Lin, and Y. Liu. 2018. Tagspin: High Accuracy Spatial Calibration of RFID Antennas via Spinning Tags. IEEE Transactions on Mobile Computing 17, 10 (Oct. 2018), 2438-2451. https://doi.org/10.1109/TMC.2018.2796092

[7] Martin Ester, Hans-Peter Kriegel, Jörg Sander, and Xiaowei Xu 1996. A Density-based Algorithm for Discovering Clusters in Large Spatial Databases with Noise. In KDD1996: Proceedings of the Second International Conference on Knowledge Discovery and Data Mining. AAAI Press, 226-231. http://dl.acm.org/ citation.cfm?id $=3001460.3001507$

[8] Jinsong Han, Han Ding, Chen Qian, Wei Xi, Zhi Wang, Zhiping Jiang, Longfei Shangguan, and Jizhong Zhao. 2016. CBID Customer Behavior Identification System Using Passive Tags. IEEE/ACM Transactions on Networking 24, 5 (2016), 28852898.

[9] Cory Hekimian-Williams, Brandon Grant, Xiuwen Liu, Zhenghao Zhang, and Piyush Kumar. 2010. Accurate localization of RFID tags using phase difference. In RFID 2010: IEEE International Conference on RFID. IEEE, Orlando, FL, USA.

[10] IBM. 2004. Item-level RFID technology redefines retail operations with real-time, collaborative capabilities. ibm.com/industries/ retail

[11] Impinj. 2013. Low Level User Data Support. www.impinj.com

[12] James Kennedy and Russell C. Eberhart. 1995. Particle swarm optimization. In Proceedings of the IEEE International Conference on Neural Networks. 1942-1948.

13] Jia Liu, Feng Zhu, Yanyan Wang, Xia Wang, Qing feng Pan, and Lijun Chen. 2017. RF-Scanner: Shelf scanning with robotassisted RFID systems, In INFOCOM 2017: Proceedings of the 36th IEEE International Conference on Computer Communications. INFOCOM 2017: Proceedings of the 36th IEEE International Conference on Computer Communications, 1-9.
[14] Tianci Liu, Lei Yang, Xiang-Yang Li, Huaiyi Huang, and Yunhao Liu. 2015. TagBooth: Deep shopping data acquisition powered by RFID tags. In INFOCOM 2015: Proceedings of the 34th IEEE International Conference on Computer Communications. 1670-1678.

[15] Tianci Liu, Lei Yang, Qiongzheng Lin, Yi Guo, and Yunhao Liu. 2014. Anchor-free Backscatter Positioning for RFID Tags with High Accuracy. In INFOCOM 2014: Proceedings of the 33th IEEE International Conference on Computer Communications. 379-387.

[16] Lionel M. Ni, Yunhao Liu, Yiu Cho Lau, and Abhishek P. Patil. 2003. LANDMARC: indoor location sensing using active RFID. In PerCom 2003: Proceedings of the IEEE International Conference on In Pervasive Computing and Communications. 407-415.

[17] Longfei Shangguan, Zheng Yang, Alex X. Liu, Zimu Zhou, and Yunhao Liu. 2015. Relative Localization of RFID Tags Using Spatial-temporal Phase Profiling. In NSDI 2015: Proceedings of the 12th USENIX Conference on Networked Systems Design and Implementation. Berkeley, CA, USA, 251-263.

[18] Longfei Shangguan, Zheng Yang Zhenjiang Li and, Mo Li, and Yunhao Liu. 2013. OTrack: Order Tracking for Luggage in Mobile RFID Systems. In INFOCOM 2013: Proceedings of the 32nd IEEE International Conference on Computer Communications. 3066-3074.

[19] C. Wang, H. Wu, and N. F. Tzeng. 2007. RFID-based 3-D Positioning Schemes. In INFOCOM 2007: Proceedings of the 26th IEEE International Conference on Computer Communications. 1235-1243.

[20] Ge Wang, Chen Qian, Longfei Shangguan, Han Ding, Jinsong Han, Nan Yang, Wei Xi, and Jizhong Zhao. 2017. HMRL: Relative Localization of RFID Tags with Static Devices. In SECON 2017: Proceedings of the 14th Annual IEEE International Conference on Sensing Communication and Networking. San Diego, CA, USA, 1-9.

[21] Jue Wang and Dina Katabi. 2013. Dude, Where's My Card?: RFID Positioning That Works with Multipath and Non-line of Sight. In SIGCOMM 2013: Proceedings of the ACM Special Interest Group on Data Communication. ACM, New York, USA, 51-62.

[22] Lei Yang, Yekui Chen, Xiangyang Li, Chaowei Xiao, Mo Li, and Yunhao Liu. 2014. Tagoram: real-time tracking of mobile RFID tags to high precision using COTS devices. In MobiCom 2014: Proceedings of the 20th Annual International Conference on Mobile Computing and Networking. Maui, HI, USA, 237-248.

[23] Dian Zhang, Jian Ma, Quanbin Chen, and Lionel M. Ni. 2007. An RF-based System for Tracking Transceiver-free Objects. In PerCom 2007: Proceeding of the 5th Annual IEEE International Conference on Pervasive Computing and Communications. 135144.

[24] Qi Zhang, M Crisp, Richard V. Penty, and I.H. White. 2015. Reduction of Proximity Effects on UHF Passive RFID Systems by Using Tags with Polarization Diversity. IEEE Transactions on Antennas and Propagation 63, 5 (May 2015), 2264-2271. https://doi.org/10.1109/TAP.2015.2403875 